Die FMH unterstützt die nationale Grippepräventionskampagne

Auch dieses Jahr schliesst sich die FMH der Grippepräventionskampagne des Kollegiums für Hausarztmedizin (KHM) an. In Zusammenarbeit mit dem Bundesamt für Gesundheit (BAG) geht es darum, beizutragen, Morbidität und Mortalität zu senken, Ansteckungsgefahr bzw. Übertragung auf andere Menschen $\mathrm{zu}$ reduzieren und Kostensteigerungen im Gesundheitswesen einzudämmen.
Die FMH lädt die Ärzteschaft - wie auch das gesamte medizinische, paramedizinische und Pflegepersonal sowie unsere Kollegen Zahnärzte und Apotheker - ein, sich dieser Kampagne breit anzuschliessen und insbesondere die Bemühungen der Organisationen der Grundversorgerärzte, welche am 4. November 2005 ihren zweiten nationalen Grippeimpftag organisieren, zu unterstützen.

Ressort Gesundheit und Prävention der FMH

\title{
Wie soll bei der Grippeimpfung anlässlich des Grippeimpftags aufgeklärt werden?*
}

\author{
Informationen zur rechtlichen Lage und ein Angebot zur praktischen Umsetzung
}

\section{U. Grüninger}

Die Hausärztinnen und -ärzte impfen sehr viele ihrer Patientinnen und Patienten gegen Grippe. Die Frage, worüber und wie ein Patient über die Grippeimpfung aufgeklärt werden muss, ist deshalb nicht neu, aber sie wurde durch den nationalen Grippeimpftag wieder aktuell. Aufgrund der juristischen Analyse der Probleme und Konsequenzen bei einer noch so unwahrscheinlichen schweren Komplikation könnte auch einem engagierten Arzt «gschmuech» werden - zum Glück ist die Lösung aber nicht so kompliziert wie das Problem.

Der folgende Artikel fasst im Vorfeld des 2. Nationalen Grippeimpftags der Schweizer Hausärztinnen und -ärzte vom 4. November 2005 die rechtlichen Anforderungen an die Patienteninformation vor einer Grippeimpfung zusammen und bietet interessierten Kollegen/-innen eine pragmatische, juristisch sinnvolle Lösung an.

\footnotetext{
* Dieser Artikel ist ursprünglich in PrimaryCare 2005;5(32-33): 665-7 erschienen.
}

Korrespondenz:

Dr. med. Ueli Grüninger

Kollegium für Hausarztmedizin

(KHM)

Landhausweg 26

CH-3007 Bern

E-Mail: ueli.grueninger@hin.ch
Die Lösung muss nicht so kompliziert sein wie das Problem.

Steve de Shazer [1]

\section{Ausgangslage}

Die Organisationen der Grundversorger (KHM, SGAM, SGIM, SGP und FMP) planen auch für 2005 einen nationalen Grippeimpftag (am 4. November). Ziel der Aktion ist, noch nicht geimpfte Risikopersonen für einen besseren Grippeschutz zu motivieren [2]. An diesem Aktionstag können sich interessierte Personen in den teilnehmenden Arztpraxen ohne Anmeldung und gegen Barbezahlung eines Pauschalbetrages gegen Grippe impfen lassen. Die Durchführung erfolgt in der Regel durch das Praxispersonal, getrennt von der ärztlichen Sprechstunde.

\section{Problemstellung aus rechtlicher Sicht}

Die Grippeimpfung gilt als eine nebenwirkungsarme Impfung und als eine wirksame Massnahme gegen die Grippeerkrankung, wobei die Wahrscheinlichkeit schwerer Impfnebenwirkungen deutlich kleiner ist als das Risiko ernsthafter Komplikationen einer Grippe.

Trotzdem haben uns einige Kollegen ihre Bedenken mitgeteilt [3], dass aus rechtlicher Sicht die Anforderungen, z.B. des BAG [4], bezüglich Aufklärung der Impflinge speziell im Rahmen eines solchen Grippeimpftags übertrieben und deshalb nicht praktikabel seien, so dass ein Arzt Gefahr laufe, bei schweren Komplikationen wegen ungenügender Aufklärung verurteilt zu werden und infolge seiner Haftpflicht gar Konkurs zu gehen. Ein Kollege monierte, dass der Staat das juristische Risiko (Haftpflicht bei Nebenwirkungen) auf die Hausärztinnen und -ärzte abwälze, obwohl die Grippeimpfung im ureigensten Interesse des Staates für die Volksgesundheit liege. 


\section{Vorgehen zur rechtlichen Klärung}

Wir haben diese Fragen den Rechtsdiensten von FMH und BAG vorgelegt, mit der Bitte, die rechtliche Situation zu klären und die für eine Impfung am Grippeimpftag zu beachtenden Punkte auszuformulieren. Aufgrund dieser Abklärung wollten wir in Zusammenarbeit mit diesen Juristen und praktizierenden Kollegen/-innen praxisnahe definieren, wie ein Praxisinhaber am Grippeimpftag den rechtlichen Vorgaben ausreichend und unter Wahrung der Verhältnismässigkeit nachkommen kann.

\section{Lage aus rechtlicher Sicht [5]}

Grundsätzlich und formal ist die rechtliche Situation bei der Grippeimpfung an einem Grippeimpftag nicht anders als bei einer Impfung bei anderer Gelegenheit, also im normalen Sprechstundenalltag, und wie generell bei der ärztlichen Arbeit: Der Arzt erfüllt einen Auftrag des Patienten, und wenn er dies nach den üblichen Regeln der beruflichen Sorgfalt tut und auch dokumentiert hat, dann hat er seinen Pflichten Genüge getan und hat auch keine rechtlichen Folgen zu befürchten, wenn sich eine Komplikation einstellen sollte. Zwei Dinge sind also zu beachten: Aufklärung ist Teil der beruflichen Sorgfaltspflicht, und im Zweifelsfall muss die erfolgte Aufklärung belegt werden können.

Die Grippeimpfung erfolgt also - wie alle Impfungen, die nicht gesetzlich zwingend vorgeschrieben sind - im Rahmen eines privatrechtlichen Auftrags des Patienten an den Arzt. Bei fehlerhaftem Verhalten haftet in erster Linie der Arzt. Im Falle von Impfschäden kommt bei behördlich angeordneten oder empfohlenen Impfungen eine subsidiäre Haftung der Kantone zum Tragen. Eine Entschädigungspflicht der Kantone fällt demnach erst in Betracht, wenn keine ausreichende Deckung von primär Ersatzpflichtigen (Arzt/Berufshaftpflichtversicherung, Sozialversicherungen) erlangt werden kann. Dabei haftet der Kanton nur in dem Umfang, der nach Inanspruchnahme anderer Ersatzpflichtiger verbleibt (sogenannte Ausfalldeckung).

Zwar hat das BAG aus Gründen der öffentlichen Gesundheit ein Interesse daran, dass die Grippeimpfung durchgeführt wird, aber es kann den Ärzten ihre Verantwortung für die korrekte Aufklärung des Patienten und die sachgerechte Durchführung der Impfung nicht abnehmen. Jedoch stellt es - im Sinne einer Hilfeleistung für die impfenden Ärzte - qualitativ hochstehende staatliche Informationsmittel bereit (Fachbro- schüre für medizinisches Personal, Informationsbroschüren für Patientinnen und Patienten).

Aus juristischer Sicht wird das Mass der geforderten Aufklärung immer durch die Besonderheiten des Einzelfalles bestimmt, weshalb nicht pauschal gesagt werden kann, welche Elemente das Aufklärungsgespräch konkret beinhalten muss. Eine angemessene Aufklärung im Rahmen des Grippeimpftages sollte i.d.R. zumindest folgende Bereiche abdecken: Fragen zum allgemeinen Gesundheitszustand des Patienten (im Hinblick auf die Impfung), Auskünfte über die beabsichtigte Massnahme (Grippeimpfung), die damit verbundenen Risiken (mögliche Nebenwirkungen usw.) sowie über Kosten und Bezahlungsweise. Bei den Richtlinien im Supplementum XVI des BAG handelt es sich um allgemeine Empfehlungen an die impfenden Ärzte; den Besonderheiten einer Grippeimpfung im Rahmen des Grippeimpftages kann Rechnung getragen werden.

\section{Information und Dokumentation praktisch}

Bei der sinngemässen Interpretation und sinnvollen Umsetzung sind also zwei Aspekte wichtig:

- der Informationsaustausch mit Impfwilligen über die Grippeimpfung (bestehende Kontraindikationen, Aufklärung über zu erwartenden Schutz und mögliche Nebenwirkungen, Bezahlung);

- die Dokumentation der durchgeführten Aufklärung und Risikoabklärung.

Diese Aufklärung und Dokumentation können ebenso wie die Grippeimpfung sehr wohl durch nichtärztliches Praxispersonal durchgeführt werden, vorausgesetzt, dass es durch den Praxisinhaber entsprechend instruiert worden ist. Was zählt ist, dass der ganze Vorgang adäquat durchgeführt und auch dokumentiert wird.

Anders gesagt: eine Impfung am Grippeimpftag unterscheidet sich nicht von einer anderen medizinischen Aktivität in der Praxis. Der Arzt und Praxisinhaber ist stets für das Geschehen in seiner Praxis verantwortlich und hat ein Interesse, dass er und seine Mitarbeiter/innen Information, Aufklärung und Durchführung entsprechend der beruflichen Sorgfalt ausüben.

Wer das nicht tut, trägt das Restrisiko, dass ein Patient mit einer - im Falle der Grippeimpfung statistisch zwar sehr seltenen - schweren Nebenwirkung ein Haftpflichtverfahren einleiten kann. 
Informationen zum 2. Nationalen Grippeimpftag der Schweizer Hausärztinnen und -ärzte vom 4. November 2005 erhalten Sie über die Korrespondenzadresse sowie auf der Website des Kollegiums für Hausarztmedizin www.kollegium.ch
Wie der Praxisinhaber seine Sorgfaltspflicht konkret umsetzen will, liegt in seinem eigenen Ermessen.

\section{Ein Dokumentationsblatt als Angebot}

Für den Fall des Grippeimpftags, und speziell für Impfungen von Personen, welche nicht schon zum bekannten Patientenstamm des impfenden Arztes gehören, bietet das KHM interessierten Kollegen/-innen eine mögliche Umsetzungshilfe in Form eines Dokumentationsblatts (eine Seite A4) an, das den obenerwähnten Anforderungen entspricht (Abb. 1).

Das KHM hat dieses einfache «Dokumentationsblatt Grippeimpftag» in Zusammenarbeit mit FMH und BAG und in Anlehnung an die Patientenbroschüre des BAG entwickelt. Auf diesem Blatt sind die vier hauptsächlichen Bereiche aufgeführt, welche aus medizinischen und rechtlichen Gründen zur Aufklärung vor einer Grippeimpfung gehören: Erfassung von Kontraindikationen, erfolgte Aufklärung anhand der BAG-Broschüre, Einverständnis des Patienten und verwendeter Impfstoff [6].

Der Ablauf kann in der Praxis wie folgt aussehen: Den Impfinteressentinnen und -interessenten wird zuerst die Impfbroschüre des BAG zum Lesen abgegeben, dann benutzt die impfende Person (also am Grippeimpftag in der Regel die MPA) das Dokumentationsblatt einerseits als Checkliste für Information und Erfassung der Kontraindikationen und andererseits zur Dokumentation des erfolgten Impfgesprächs (fünf Kästchen zum Ankreuzen, Praxisvisum).

Dieses Dokumentationsblatt wird auf der Website des Kollegiums für Hausarztmedizin (www.kollegium.ch) und auf der Grippewebsite des BAG (www.grippe.admin.ch) zum Download zur Verfügung stehen. Das Dokumentationsblatt ist Teil der Sammlung der für den Grippeimpftag angebotenen Hilfsmittel, und dessen Benützung ist wie bereits gesagt freiwillig.

\section{Schlussbemerkung: Tempora mutantur oder auf dem schmalen Grat zwischen Verhältnismässigkeit und Zeitgeist}

Viele von uns befragte Hausärztinnen und -ärzte haben die eingangs geschilderten rechtlichen Bedenken einiger Kolleginnen und Kollegen als unberechtigt oder übertrieben bezeichnet und bedauernd betont, dass mit solchen Anfragen eine juristische Pandorabüchse geöffnet werde, wodurch letztlich eine realistische ärztliche Arbeit verunmöglicht werden könnte. Diese Bedenken sind auf dem Hintergrund einer traditionellen, arztzentrierten Praxisführung verständlich, und die Frage der Grenzen der Verhältnismässigkeit stellt sich tatsächlich.

Andererseits haben sich die Zeiten und damit die gesellschaftlichen Anforderungen an unsere ärztliche Rechenschaftspflicht geändert, und das hat Folgen - dies zeigt sich ja gerade daran, dass die Leserbriefschreiber eine haftpflichtrechtliche Klage und Verurteilung als reale Möglichkeit und neue Art der Bedrohung erleben.

Dieses Dilemma ist real und auch nicht neu, der Grippeimpftag hat es nur wieder neu thematisiert. Dieses Dilemma zu verwünschen, zu verleugnen oder davor die Augen zu verschliessen, würde nichts bringen: Wir müssen akzeptieren, dass wir bei der ärztlichen Arbeit auf dem schmalen Grat zwischen praktischer Verhältnismässigkeit und rechtlicher Risikominimierung unsere Schritte bewusst setzen müssen. Das geht mit offenen Augen nicht unbedingt leichter, aber besser. Und wie gesagt: Zum Glück sind sich Grundversorger/innen das Leben mit einem Rest an Ungewissheit gewöhnt.

\section{Literatur}

1 de Shazer S. Wege der erfolgreichen Kurztherapie. Stuttgart: Klett; 1989

2 Zu diesen Risikogruppen gehören: Menschen über 65, Personen mit chronischen Erkrankungen, Heimbewohner/innen, Medizinal- und Pflegepersonal sowie Kontaktpersonen, die Risikopersonen anstecken könnten (vgl. PrimaryCare 2005;5(17):388-9 sowie Schweiz Ärztezeitung 2005;86(17):1009).

3 Leserbriefe König (PrimaryCare 2005;5[10]:221) und Schiller (PrimaryCare 2005;5[17]:392), ferner einzelne Briefe sowie Kommentare in Evaluationsumfrage zum Grippeimpftag 2004.

4 «Allgemeine Empfehlungen zu Impfungen», im Supplementum XVI zum blauen Ordner Infektionskrankheiten des BAG, Abschnitt Rechtsfragen (Absätze: Impfvisite, Einwilligung, Anamnese, Impfung und Dokumentation).

5 Der Autor dankt den Fürsprechern Thomas Bertschy vom Rechtsdienst des BAG und Hanspeter Kuhn vom Rechtsdienst der FMH für ihre wertvolle Mitarbeit bei der Klärung der rechtlichen und formalen Anforderungen für Patienteninformation und -aufklärung.

6 Die Medizinische Gesellschaft Basel MedGes hat übrigens für ihre Grippeimpfaktion 2005 ebenfalls einen Fragebogen, insbesondere für die Erfassung von Kontraindikationen, entwickelt, wie wir erst nach Abschluss unserer Arbeiten erfahren haben (www.medges.ch). 
Abbildung 1

Dokumentationsblatt Grippeimpftag. Download von: www.kollegium.ch oder www.grippe.admin.ch.
KHM CMPR CMB
KOLEGIUMER HAUSARTMEDIZN
COLEGE DE MEDECINE DEREMER RECURS
Dokumentationsblatt Grippeimpftag 2005
Name:
Vorname:
Jahrgang:
Adresse:
Ort:

\section{Kontraindikationen}
- Haben Sie gegenwärtig Fieber?
Nein Ja
- Haben Sie eine Überempfindlichkeit auf Hühnereiweiss?
$\square \quad \square$ später impfen
- Sind bei früheren Impfungen Probleme aufgetreten?
$\square$ mit Arzt besprechen
mit Arzt besprechen

Information der Patientin/des Patienten über die Grippeimpfung:

Die Patientenbroschüre des Bundesamtes für Gesundheit (Faltprospekt zur Grippeimpfung) wurde abgegeben und folgende Punkte besprochen:

- Wovor schützt die Grippeimpfung?

- Welche möglichen Nebenwirkungen ${ }^{1}$ resp. welches Risiko ${ }^{2}$ beinhaltet die Grippeimpfung?

- Wer soll sich impfen lassen?

- Wer bezahlt?

\section{Auftrag der Patientin/des Patienten}

Will die Grippeimpfung durchführen

Ja

Verwendeter Impfstoff:

Datum des Impfgesprächs $\quad$ Visum Arztpraxis

${ }^{1}$ Rötungen an der Impfstelle, Schmerzen und Jucken, selten leichtes Fieber und Übelkeit sowie Muskelschmerzen; ganz selten können Nesselsucht, Schwellungen, allergisches Asthma oder eine allergische Sofortreaktion auftreten.

${ }^{2}$ Das Risiko ernsthafter Komplikationen nach einer Grippe ist höher als die Wahrscheinlichkeit schwerer Nebenwirkungen nach der Impfung. 